\title{
Successful Treatment with Noninvasive Positive-pressure Ventilation Based on the Prediction of Disease Onset Using CT and Respiratory Function Tests in an Elderly Patient with Relapsing Polychondritis
}

\author{
Hiromichi Yamaguchi, Yuko Komase, Ayami Ono, Akane Morita and Akira Ishida
}

\begin{abstract}
An 83-year-old man who had been receiving treatment for bronchial asthma since 62 years of age experienced difficulty breathing on exertion and was admitted to the hospital. On admission, computed tomography revealed tracheal wall thickening, while test results for antinuclear antibodies and anti-type II collagen antibodies were positive. Since a saddle nose deformity, malacia of the auricles and sensorineural deafness were also observed, relapsing polychondritis was diagnosed. Measuring the peak expiratory flow rate was useful in the early airway assessment. During the follow-up period, the patient's dyspnea worsened and noninvasive positive-pressure ventilation was introduced. As a result, the subjective symptoms improved.
\end{abstract}

Key words: relapsing polychondritis, bronchial asthma, noninvasive positive-pressure ventilation, anti-type II collagen antibody, respiratory function tests, PEFR

(Intern Med 52: 1085-1089, 2013)

(DOI: 10.2169/internalmedicine.52.9035)

\section{Introduction}

Relapsing polychondritis (RP) is a chronic inflammatory disease of unknown etiology, the diagnostic criteria of which were proposed by McAdam et al. in 1976. Anti-type II collagen antibodies are often found in the sera of patients with $\mathrm{RP}$, thus indicating that autoimmune abnormalities are associated with this disease (1). The mean age at onset is approximately 50 years; however, adolescent and elderly individuals (70 years of age or older) can also develop the disease (2). Since patients with RP present with expiratory wheezing and respiratory function tests that show obstructive ventilatory impairment, RP is easily misdiagnosed as asthma. Therefore, making a relatively early diagnosis of RP is difficult. Although RP is diagnosed in many patients after they receive treatment for asthma, relatively few studies have reported asthma and RP as comorbidities. A major non-pharmacological treatment of RP is tracheal stent placement, and some reports show improvement of the respiratory function with continuous positive airway pressure
(CPAP) $(3,4)$.

We encountered an interesting case of RP in which (1) the patient had a 20-year history of asthma and developed $\mathrm{RP}$ at 83 years of age, (2) the patient tested positive for anti-type II collagen antibodies, (3) the onset of RP was predicted using respiratory function tests and computed tomography (CT), and the peak expiratory flow rates (PEFR) calculated from flow-volume curves were useful for early airway assessment, and (4) the introduction of bilevel positive airway pressure (PAP) therapy improved the patient's subjective symptoms. We describe this case with a review of the relevant literature.

\section{Case Report}

The patient was an 83-year-old man who had been receiving treatment for bronchial asthma (atopic and severely persistent) since 1989. Notwithstanding good asthma control, he experienced difficulty breathing starting in December 2009. Despite increased dosages of steroids and omalizumab given to treat the asthma, the patient's symptoms did not 


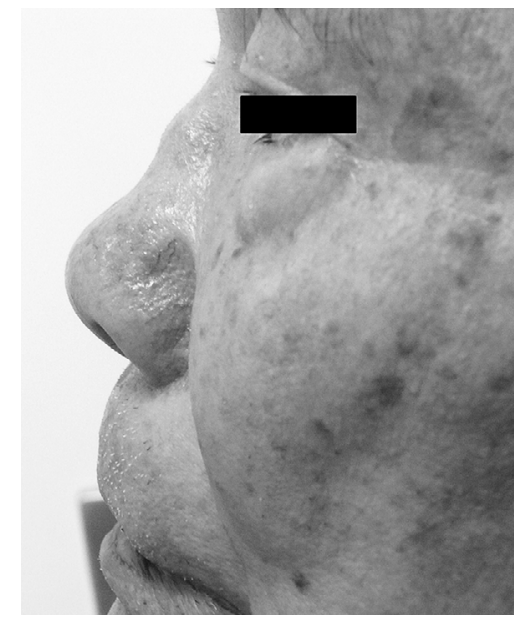

Figure 1. On admission, a saddle nose deformity was observed.

improve, and he was admitted to the hospital on November, 2010 for a detailed examination and treatment. On admission, his blood pressure was $124 / 76 \mathrm{mmHg}$, his heart rate was 100 beats/min, his respiratory rate was 24 breaths/min and his $\mathrm{SpO}_{2}$ was $97 \%$ on room air. A physical examination revealed a saddle nose deformity and malacia of the auricles (Fig. 1). Mild rhonchi were heard on expiration. In addition, the patient complained of tinnitus and hearing loss, and an otolaryngologist had diagnosed sensorineural deafness. Laboratory tests performed on admission revealed a 1,280fold increase in antinuclear antibodies and positive anti-type II collagen antibodies $(78.0 \mathrm{EU} / \mathrm{mL})$. The levels of other inflammatory markers were normal. On respiratory function tests, the patient exhibited marked obstructive impairment: the forced vital capacity (FVC), PEFR and forced expiratory volume in 1 second $\left(\mathrm{FEV}_{1}\right)$ had decreased since September 2009 (Fig. 2). After September 2009 when further decreases in $\mathrm{FEV}_{1}$ occurred, the lack of a reversible airway obstruction response suggested that the declined $\mathrm{FEV}_{1}$ was due to additive airway obstruction, not to worsened asthma. Chest CT performed on admission revealed tracheal and bronchial wall thickening and luminal narrowing. No airway thickening was observed on previous CT images obtained in May 2009 (Fig. 3A, B, C), although it was observed in January 2010 (Fig. 3D, E, F). Based on the imaging and facial findings, the increased levels of antinuclear antibodies and the positive test results for anti-type II collagen antibodies, RP was diagnosed. A bronchoscopic examination revealed narrowing of the tracheal lumen (Fig. 4). After admission, increased doses of steroids were administered to treat the asthma, and the patient's dyspnea improved. Subsequently, he was discharged and followed up as an outpatient. In January 2011, his dyspnea worsened, and noninvasive positive pressure ventilation (NPPV) was introduced. With the inspiratory positive airway pressure (IPAP) set at $8 \mathrm{~cm} \mathrm{H}_{2} \mathrm{O}$, the expiratory positive airway pressure (EPAP) set at $4 \mathrm{~cm} \mathrm{H}_{2} \mathrm{O}$ and the respiratory rate set at 15 breaths/min, NPPV was used at night and during the daytime when the symptoms of dysp- nea appeared. Consequently, the patient's daytime dyspnea improved, and the mean nocturnal oxygen saturation increased from $95 \%$ to $97 \%$ after the introduction of NPPV. The patient again experienced difficulty breathing in November 2011 when he developed an infection, and the NPPV settings were changed as follows: IPAP to $12 \mathrm{~cm}$ $\mathrm{H}_{2} \mathrm{O}$, EPAP to $6 \mathrm{~cm} \mathrm{H}_{2} \mathrm{O}$ and the respiratory rate to 12 breaths/min (Fig. 2). The patient's symptoms did not subsequently worsen, and he has since been followed as an outpatient.

\section{Discussion}

The currently used diagnostic criteria for RP were initially proposed by McAdam et al. in 1976 (5) and later revised by Damiani et al. in 1979 (6). According to the criteria, obtaining histological confirmation of RP is not necessary if the patient has three or more of the following conditions: 1) bilateral auricular relapsing chondritis; 2) nasal chondritis; 3) ocular inflammation; 4) non-erosive inflammatory arthritis; 5) respiratory tract chondritis; and 6) cochlear and/or vestibular damage. Among patients with RP, 22-66\% test positive for antinuclear antibodies, $16 \%$ test positive for rheumatoid factor (RF) (7-9) and approximately 30-70\% test positive for autoantibodies against type II collagen, a component of cartilage (1), thus indicating that some autoimmune abnormalities are associated with the disease. Antitype II collagen antibodies are also detected in approximately $15 \%$ of patients with chronic arthritis. However, the anti-type II collagen antibodies found in patients with RP are primarily antibodies to native type II collagen, suggesting that the antibodies themselves are directly involved in the onset of RP (1). Our patient tested positive for antinuclear antibodies and anti-type II collagen antibodies and presented with deformation of the tracheal cartilage, a saddle nose deformity, auricular deformation and sensorineural deafness, which led to the diagnosis of RP.

It has been reported that half of patients with RP present with airway lesions during the course of the disease, with 5and 10-year survival rates of $74 \%$ and $55 \%$, respectively (10). Airway lesions, including those that lead to respiratory failure due to airway stenosis and collapse and repeated respiratory infections, are responsible for $10-59 \%$ of deaths associated with RP (11). Therefore, airway lesions are important prognostic factors in patients with RP, and providing assessment and management of airway lesions over time is very important. Although these findings illustrate that early diagnosis and treatment are critical to the prognosis, Trentham et al. reported that the mean time from the onset of symptoms to diagnosis is 2.9 years, thus indicating that making an early diagnosis of RP is difficult (2).

The average age at onset of RP is approximately 50 years, with slightly more women being affected than men (2). Elderly individuals (70 years of age or older) are also known to develop RP. Regarding the present patient, it is particularly interesting to know whether the original diag- 


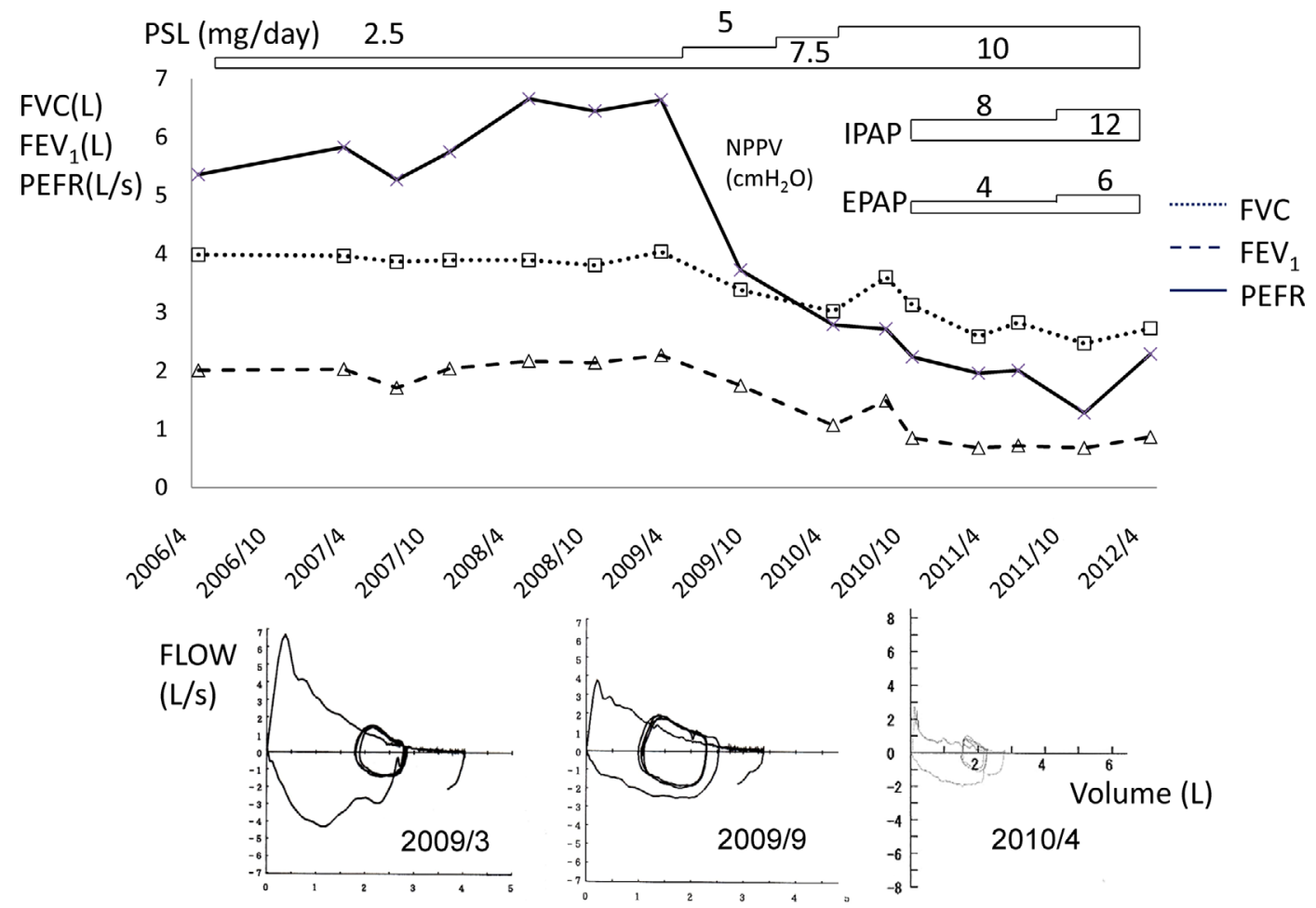

Figure 2. The patient's clinical course. EPAP: expiratory positive airway pressure, FEV $_{1}$ : forced expiratory volume in 1 second, FVC: forced vital capacity, IPAP: inspiratory positive airway pressure, NPPV: noninvasive positive pressure ventilation, PEFR: peak expiratory flow rates, PSL: prednisolone
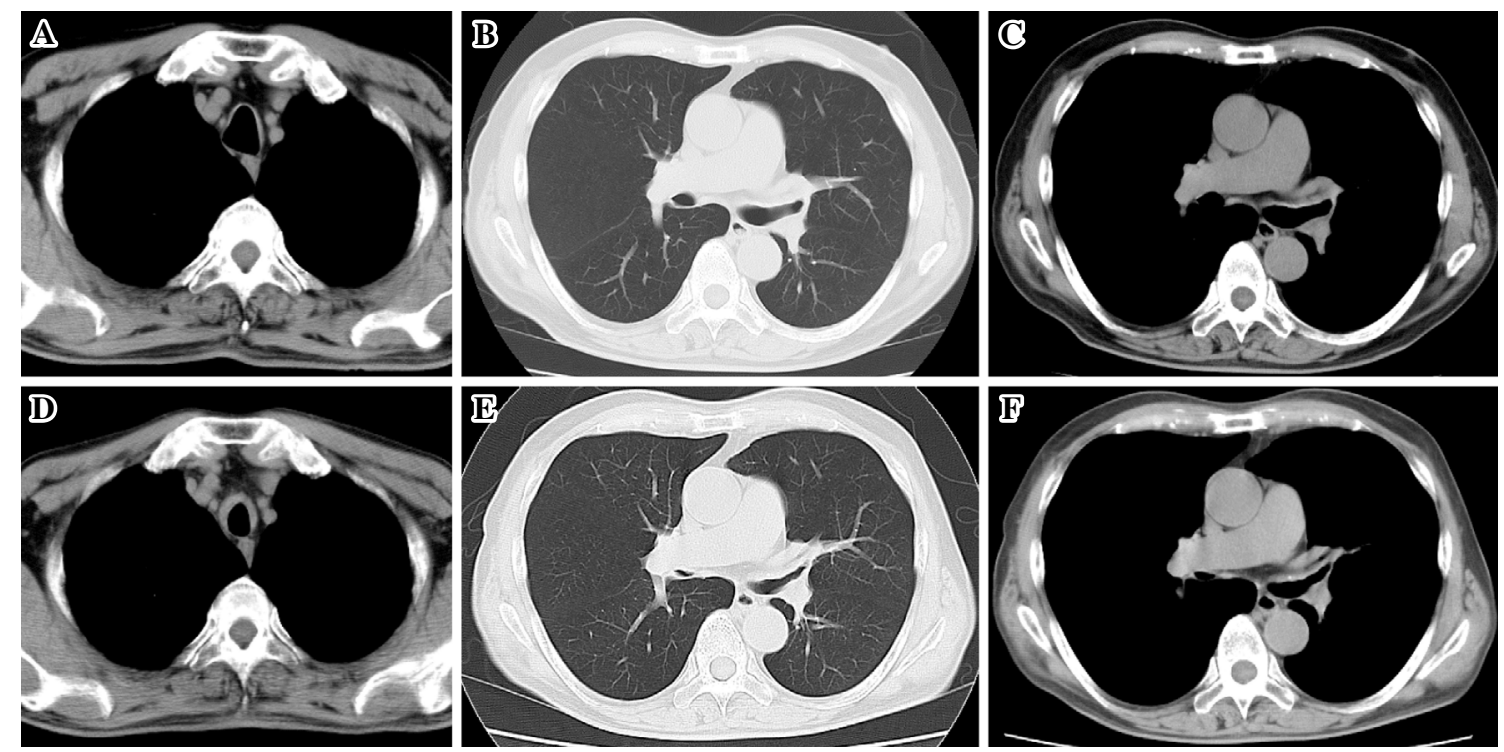

Figure 3. Chest computed tomography (CT). No tracheal wall thickening or narrowing of the bilateral principal bronchi were observed in May 2009 (A, B, C), although they were observed in January 2010 (D, E, F).

nosis of asthma was correct, and at what point in the course of his asthma did he developed RP.

Since the incidence of RP is low and patients with RP present with expiratory wheezing, many of these patients receive treatment under the diagnosis of intractable asthma or emphysema. Our patient was found to have asthma at 62 years of age, and his IgE level increased to $610 \mathrm{IU} / \mathrm{mL}$ in 1998. Reversible airway obstruction was seen in 1995 and 2005. Tests to assess airway hyperresponsiveness were not conducted in our hospital at that time. Considering these observations, it is likely that the initial diagnosis of asthma was correct. 


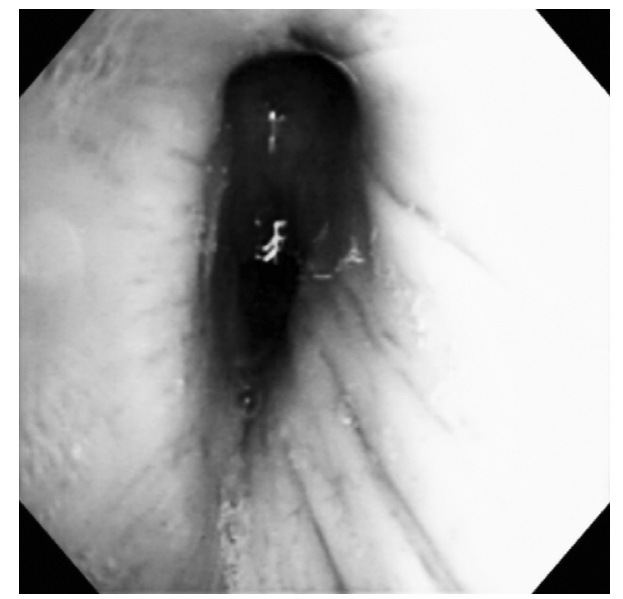

Figure 4. Bronchoscopic findings of the right principal bronchus. The mucous membranes were edematous and the lumen was narrowed.

Conducting a bronchoscopic examination is important and useful for determining the location and severity of RP airway lesions. However, the procedure is highly invasive, resulting in an increased risk of aggravation of respiratory symptoms. The benefits of other types of airway assessment, including CT (12), respiratory function tests, flow-volume curves and PEFR measurements, have also been reported $(11,13,14)$. In addition, it was recently reported that fluorodeoxyglucose positron-emission tomography (PET) is useful for diagnosing RP (15). Port et al. emphasize that CT is a useful imaging modality for making the differential diagnosis because tracheal wall thickening detected on CT scans is characteristic of RP airway lesions (16). It has also been reported that $\mathrm{CT}$ scans can identify tracheal and bronchial stenosis at relatively early stages of RP, in contrast to bronchoscopic examinations (17). Regarding the respiratory function, obstructive ventilatory impairment, including decreases in PEFR and $\mathrm{FEV}_{1}$ on flow-volume curves, is observed in patients with RP. The expiratory obstruction observed in RP may be due, not to loss of pulmonary elasticity, as found in chronic obstructive pulmonary disease (COPD), but to airway collapse during expiration, owing to a loss of supporting tissue caused by chondritis or cicatricial stenosis of the airways (14). CT images of the present patient and the results of respiratory function tests were retrospectively examined to determine the time of RP onset during the course of the patient's asthma. No tracheal wall thickening was observed on CT images in May 2009; however, it was observed on CT images starting in January 2010. As for the respiratory function, reversibility tests performed after 2006 showed no changes in $\mathrm{FEV}_{1}$ before or after inhalation of short-acting $\beta_{2}$ stimulants, and the patient seemed to have good asthma control. After April 2010, however, inhalation of short-acting $\beta_{2}$ stimulants did not improve $\mathrm{FEV}_{1}$ to the previous levels, thus suggesting that irreversible airway obstruction had developed since then. Moreover, there were sharp decreases in PEFR on flow-volume curves starting in September 2009. Based on these observations, we can conclude that the patient likely developed RP around September 2009, and the time to diagnosis was approximately one year and two months after onset. Considering the CT findings, it is possible that the tracheal wall rapidly thickened over the course of four months. The changes in the results of the respiratory function tests and the findings in CT images over time indicate that PEFR measurement on flow-volume curves may be useful for early airway assessment in patients with RP. Treatment of RP often involves medications for infections at the early stages, expectorants and drugs to reduce airway secretions. If patients have bronchial asthma, emphysema or other respiratory comorbidities, adequate treatment for the underlying disease, such as steroids, is administered in addition to treatment for symptoms $(18,19)$. Because our patient had received Step 4 therapy for severe persistent asthma (in accordance with the Asthma Prevention and Management Guidelines) and had taken steroids for a long time, the symptoms of saddle nose deformity and auricular chondritis might have been relieved, resulting in a delay in the onset of RP symptoms. For patients whose symptoms are not improved by medications, tracheal stent placement, the major non-pharmacological treatment, is often performed (18). Since stent placement for benign disease may cause granulation tissue formation and infections, the indication for the procedure should be carefully considered. However, the use of tracheotomy and intubation may be necessary for survival in patients with significant respiratory impairment. Several recent studies have reported marked improvement of the respiratory function with nasal CPAP $(3,4)$. Taking the advanced age of the patient into consideration, we first tried to improve his respiratory status by using a non-invasive respirator. Since the improvement of airway collapse achieved with CPAP alone did not seem to provide sufficient ventilator volume, bilevel PAP was applied from the beginning. Consequently, the patient's daytime subjective symptoms and nocturnal oxygen saturation improved. The pathophysiology of RP supports the idea that maintaining positive airway pressure is effective for preventing expiratory airway obstruction. Two years have passed since the introduction of NPPV in the present case, and no worsening of symptoms has been observed. Thus far, the treatment has been effective; however, stent placement would become necessary if the patient's symptoms were to worsen in the future.

In conclusion, although the importance of making the differential diagnosis between RP and asthma is widely recognized, it is necessary to consider the possibility of other comorbidities in patients with intractable asthma. In addition, the present case illustrates that NPPV is effective as a non-pharmacological treatment in elderly patients with RP.

The authors state that they have no Conflict of Interest (COI).

\section{References}

1. Foidart JM, Abe S, Martin GR, et al. Antibodies to type II colla- 
gen in relapsing polychondritis. N Engl J Med 299: 1203-1207, 1978.

2. Trentham DE, Le CH. Relapsing polychondritis. Ann Intern Med 129: 114-122, 1998.

3. Carden KA, Boiselle PM, Waltz DA, Ernst A. Tracheomalacia and tracheobronchomalacia in children and adults: an in-depth review. Chest 127: 984-1005, 2005.

4. Ferguson GT, Benoist J. Nasal continuous positive airway pressure in the treatment of tracheobronchomalacia. Am Rev Respir Dis 147: 457-461, 1993.

5. McAdam LP, O'Hanlan MA, Bluestone R, Pearson CM. Relapsing polychondritis: prospective study of 23 patients and a review of the literature. Medicine 55: 193-215, 1976.

6. Damiani JM, Levine HL. Relapsing polychondritis--report of ten cases. Laryngoscope 89: 929-946, 1979.

7. Piette JC, El-Rassi R, Amoura Z. Antinuclear antibodies in relapsing polychondritis. Ann Rheum Dis 58: 656-657, 1999.

8. Balsa-Criado A, Gonzalez-Hernandez T, Cuesta MV, Aguado P, Garcia S, Gijon J. J Lupus anticoagulant in relapsing polychondritis. Rheumatol 17: 1426-1427, 1990.

9. Michet CJ. Vasculitis and relapsing polychondritis. Rheum Dis Clin North Am 16: 441-444, 1990.

10. Michet CJ Jr, McKenna CH, Luthra HS, O'Fallon WM. Relapsing polychondritis. Survival and predictive role of early disease mani- festations. Ann Intern Med 104: 74-78, 1986.

11. Sarodia BD, Dasgupta A, Mehta AC. Management of airway manifestations of relapsing polychondritis: case reports and review of literature. Chest 116: 1669-1675, 1999.

12. Port JL, Khan A, Barbu RR. Computed tomography of relapsing polychondritis. Comput Med Imaging Graph 17: 119-123, 1993.

13. Mohsenifar Z, Tashkin DP, Carson SA, Bellamy PE. Pulmonary function in patients with relapsing polychondritis. Chest 81: 711717, 1982.

14. Krell WS, Staats BA, Hyatt RE. Pulmonary function in relapsing polychondritis. Am Rev Respir Dis 133: 1120-1123, 1986.

15. De Geeter F, Vandecasteele SJ. Fluorodeoxyglucose PET in relapsing polychondritis. N Engl J Med 358: 536-537, 2008.

16. Port JL, Khan A, Barbu RR. Computed tomography of relapsing polychondritis. Comput Med Imaging Graph 17: 119-123, 1993.

17. Tillie-Leblond I, Wallaert B, Leblond D, et al. Respiratory involvement in relapsing polychondritis. Clinical, functional, endoscopic, and radiographic evaluations. Medicine 77: 168-176, 1998.

18. Segel MJ, Godfrey S, Berkman N. Relapsing polychondritis: reversible airway obstruction is not always asthma. Mayo Clin Proc 79: 407-409, 2004.

19. Lipnick RN, Fink CW. Acute airway obstruction in relapsing polychondritis: treatment with pulse methylprednisolone. J Rheumatol 18: 98-99, 1991.

(C) 2013 The Japanese Society of Internal Medicine http://www.naika.or.jp/imonline/index.html 